\title{
UNIT ORTHODOX SEMIGROUPS
}

\author{
by T. S. BLYTH and R. McFADDEN $\dagger$
}

(Received 5 May, 1981)

Let $S$ be a regular semigroup. Given $x \in S$, we shall say that $a \in S$ is an associate of $x$ if $x a x=x$. The set of associates of $x \in S$ will be denoted by $A(x)$. Now suppose that $S$ has an identity element 1 . Let $H_{1}$ denote the group of units of $S$. Then we say that $u \in S$ is a unit associate of $x$ whenever $u \in A(x) \cap H_{1}$. In what follows we shall write $U(x)=$ $A(x) \cap H_{1}$ and we shall say that $S$ is unit regular $[1,3]$ if $(\forall x \in S) U(x) \neq \varnothing$. Examples of unit regular semigroups include the full transformation semigroup on a finite set [1] and the semigroup of endomorphisms of a finite-dimensional vector space [3]. In this paper we shall be concerned with semigroups that are unit orthodox (i.e. unit regular and orthodox), and we shall describe completely the structure of those semigroups that are uniquely unit orthodox (i.e. orthodox and uniquely unit regular in the sense that, for every $x \in S$, the set $U(x)$ is a singleton). It is worthy of mention that neither of the examples cited above is of this type.

We begin by examining the sets of unit associates.

THEOREM 1. Let $S$ be a unit orthodox semigroup. If $x \in S$ then

$$
u, v, w \in U(x) \Rightarrow u v^{-1} w \in U(x) .
$$

Proof. If $u, v, w \in U(x)$ then it is readily seen that $v x w$ and $w x u$ are inverses of $x$. Denoting by $Y$ the finest inverse semigroup congruence on $S$ we therefore have, $S$ being orthodox, that $v x w \mathscr{y} w x u$ whence $x w y v^{-1} w x u$. Now since $x w$ is idempotent and $y$ is idempotent-determined it follows that $v^{-1} w x u$ is idempotent, so that

$$
v^{-1} w x u \cdot v^{-1} w x u=v^{-1} w x u
$$

whence, by cancellation, we obtain $u v^{-1} w \in U(x)$.

Corollary 1. For every $x \in S, U(x)$ is a coset of some subgroup of $H_{1}$.

Proof. This is immediate from Theorems 22 and 23 of [2], which together show that a non-empty subset $X$ of a group $G$ is a coset if and only if $X=X X^{-1} X$.

Corollary 2. If $e \in S$ is idempotent then $U(e)$ is a subgroup of $H_{1}$.

Proof. Taking $x=e$ in the theorem we can choose $w=1$.

If $u \in U(x)$ then $x u$ is idempotent and so $U(x u)$ is a subgroup of $H_{1}$. It is instructive to see how $U(x)$ can be expressed as a coset of this subgroup.

THEOREM 2. Let $S$ be a unit orthodox semigroup. Given $x \in S$, we have that

$$
(\forall u \in U(x)) \quad U(x)=u U(x u) .
$$

$\dagger$ Honorary Visiting Professor at the University of St. Andrews, 1980-81.

Glasgow Math. J. 24 (1983) 39-42. 
Proof. Let $u, v \in U(x)$. Then since $x u . u^{-1} v . x u=x u$ we have $u^{-1} v \in U(x u)$ whence $v \in$ $u U(x u)$ and consequently $U(x) \subseteq u U(x u)$. But if $w \in U(x u)$ then $x u . w . x u=x u$ gives $x u w x=x$ so that $u w \in U(x)$. Thus we have the converse inclusion $u U(x u) \subseteq U(x)$.

The above observations may be used to obtain a simple necessary and sufficient condition for a unit orthodox semigroup to be uniquely unit orthodox:

Theorem 3. A unit orthodox semigroup $S$ is uniquely unit orthodox if and only if, for every idempotent $e$, the subgroup $U(e)$ is trivial.

Proof. The condition is clearly necessary. Suppose, conversely, that $U(e)=\{1\}$ for every idempotent $e$. Given $x \in S$, let $u, v \in U(x)$. By Theorem 2 we have $u U(x u)=U(x)=$ $v U(x v)$ whence, since $x u$ and $x v$ are idempotents, we deduce that $u=v$.

We recall now that if $S$ and $T$ are semigroups and if $\zeta: T \rightarrow \operatorname{Aut}(S)$, described by $x \mapsto \zeta_{x}$, is a contravariant morphism from $T$ to the group of automorphisms on $S$ then the semidirect product (relative to $\zeta$ ) of $S$ and $T$ is the semigroup $S \times{ }_{\zeta} T$ consisting of the set $S \times T$ equipped with the law of composition

$$
(a, x)(b, y)=\left(a . b \zeta_{x}, x y\right)
$$

[Note. The contravariance of $\zeta$ is required when writing the mappings $\zeta_{x}$ on the right.]

We now show how the notion of semidirect product can be used to provide a method of constructing uniquely unit orthodox semigroups.

THEOREM 4. Let $E$ be $a$ band with an identity element and let $G$ be a group. Then for every contravariant morphism $\zeta: G \rightarrow \operatorname{Aut}(E)$ the semidirect product $E \times{ }_{\zeta} G$ is a uniquely unit orthodox semigroup whose band of idempotents is isomorphic to $E$ and whose group of units is isomorphic to $G$.

Proof. We shall write, without confusion, the identity elements of $G$ and $E$ as simply 1. Describing $\zeta$ by $x \mapsto \zeta_{x}$ we observe that $\zeta_{1}=\mathrm{id}_{E}$. We also note that, for every $x \in G$ and every $e \in E$,

$$
\begin{aligned}
e \cdot 1 \zeta_{x}=e \zeta_{1} \cdot 1 \zeta_{x} & =e \zeta_{x x^{-1}} \cdot 1 \zeta_{x}=e \zeta_{x-1} \zeta_{x} \cdot 1 \zeta_{x} \\
& =\left(e \zeta_{x}-1 \cdot 1\right) \zeta_{x}=e \zeta_{x}-1 \zeta_{x}=e \zeta_{1}=e
\end{aligned}
$$

and similarly $1 \zeta_{x} . e=e$, whence $1 \zeta_{x}=1$. It now follows that $(1,1)$ is the identity element of $E \times{ }_{\zeta} G$; for

$$
\begin{aligned}
& (e, x)(1,1)=\left(e \cdot 1 \zeta_{x}, x\right)=(e, x) \\
& (1,1)(e, x)=\left(1 . e \zeta_{1}, x\right)=(e, x)
\end{aligned}
$$

Now if $(e, x)$ is a unit in $E \times{ }_{\zeta} G$ then it follows that $e$ is a unit in the band $E$ and so $e=1$; and conversely, every element of the form $(1, x)$ is a unit of $E \times{ }_{\zeta} G$ with $(1, x)^{-1}=\left(1, x^{-1}\right)$. Thus the set of units of $E \times{ }_{\zeta} G$ is $\{(1, x) ; x \in G\}$ and this is clearly isomorphic to the group $G$ under the assignment $x \mapsto(1, x)$. 
If now $(e, x)$ is an idempotent then obviously $x=1$; and conversely $(e, 1)(e, 1)=$ $\left(e . e \zeta_{1}, 1\right)=\left(e^{2}, 1\right)=(e, 1)$. Since $(e, 1)(f, 1)=(e f, 1)$ it is now clear that the mapping described by $e \mapsto(e, 1)$ is an isomorphism from $E$ to the band of idempotents of $E \times{ }_{\zeta} G$.

Finally, the fact that

$$
(e, x)\left(1, x^{-1}\right)(e, x)=\left(e .1 \zeta_{x}, 1\right)(e, x)=(e, 1)(e, x)=(e, x)
$$

shows that $E \times{ }_{5} G$ is unit orthodox; and

$$
(e, x)(1, y)(e, x)=(e, x) \Leftrightarrow y=x^{-1}
$$

so that $E \times{ }_{\zeta} G$ is uniquely unit orthodox.

That every uniquely unit orthodox semigroup can be obtained in the above way is established in the following result.

THEOREM 5. Let $S$ be a uniquely unit orthodox semigroup with band of idempotents $E$. For every $a \in S$ let $U(a)=\left\{u_{a}\right\}$; and for every $u \in H_{1}$ let $\zeta_{u}: E \rightarrow E$ be described by $e \zeta_{u}=u e u^{-1}$. Then $u \mapsto \zeta_{u}$ describes a contravariant morphism $\zeta: H_{1} \rightarrow \operatorname{Aut}(E)$ and $S \cong$ $E \times_{5} H_{1}$ under the assignment $a \mapsto\left(a u_{a}, u_{a}^{-1}\right)$.

Proof. We note first that, for every $u \in H_{1}$, the mapping $\zeta_{u}$ is clearly injective and a morphism. It is also surjective since for every $f \in E$ we have $u^{-1} f u \in E$ with $\left(u^{-1} f u\right) \zeta_{u}=f$. Thus $\zeta_{u} \in \operatorname{Aut}(E)$ for every $u \in H_{1}$. That $\zeta: H_{1} \rightarrow \operatorname{Aut}(E)$ described by $u \mapsto \zeta_{u}$ is a contravariant morphism is readily seen. We can therefore construct the semidirect product $E \times{ }_{b} H_{1}$. Now since for every $a \in S$ we have $U(a)=\left\{u_{a}\right\}$, it follows that $a u_{a} \in E$ and we can consider the mapping $\theta: S \rightarrow E \times{ }_{b} H_{1}$ described by $a \theta=\left(a u_{a}, u_{a}^{-1}\right)$. Since

$$
\left(a u_{a}, u_{a}^{-1}\right)=\left(b u_{b}, u_{b}^{-1}\right) \Rightarrow a=a u_{a} \cdot u_{a}^{-1}=b u_{b} \cdot u_{b}^{-1}=b
$$

we have that $\theta$ is injective. To see that $\theta$ is also surjective, let $(e, x) \in E \times{ }_{5} H_{1}$. Then we deduce from $e x \cdot x^{-1} \cdot e x=e x$ that $u_{e x}=x^{-1}$, so

$$
(e x) \theta=\left(e x . u_{e x}, u_{e x}^{-1}\right)=\left(e x \cdot x^{-1}, x\right)=(e, x) .
$$

Finally, we show that $\theta$ is a morphism. For this purpose, we note that $a=a u_{a} a$ implies that $u_{a} a u_{a}$ is an inverse of $a$. Since $S$ is orthodox, $u_{b} b u_{b} \cdot u_{a} a u_{a}$ is then an inverse of $a b$. Thus

$$
a b=a b \cdot u_{b} b u_{b} u_{a} a u_{a} \cdot a b=a \cdot b u_{b} b \cdot u_{b} u_{a} \cdot a u_{a} a \cdot b=a b \cdot u_{b} u_{a} \cdot a b
$$

and hence $u_{b} u_{a} \in U(a b)$. But $U(a b)=\left\{u_{a b}\right\}$ so we have that $u_{b} u_{a}=u_{a b}$. Using this we now see that

$$
\begin{aligned}
a \theta . b \theta & =\left(a u_{a}, u_{a}^{-1}\right)\left(b u_{b}, u_{b}^{-1}\right) \\
& =\left(a u_{a} .\left(b u_{b}\right) \zeta_{u_{a}^{-1}}, u_{a}^{-1} u_{b}^{-1}\right) \\
& =\left(a u_{a} u_{a}^{-1} b u_{b} u_{a}, u_{a}^{-1} u_{b}^{-1}\right) \\
& =\left(a b u_{b} u_{a},\left(u_{b} u_{a}\right)^{-1}\right) \\
& =\left(a b u_{a b}, u_{a b}^{-1}\right) \\
& =(a b) \theta . \quad \square
\end{aligned}
$$


REMARK. In Theorem 5 we proved that if $S$ is uniquely unit orthodox then for all $a, b \in S$ we have $u_{a b}=u_{b} u_{a}$. Conversely, this property implies that $S$ is orthodox; for if $e, f$ are idempotents then

$$
e f=e f u_{e f} e f=e f u_{f} u_{e} e f=e f .1 .1 . e f=(e f)^{2} .
$$

\section{REFERENCES}

1. H. D'Alarcao, Factorizable as a finiteness condition, Semigroup Forum 20 (1980), 281-282.

2. P. Dubreil, Contribution à la théorie des demi-groupes, Mém. Acad. Sci. Inst. France (2) 63 (1941), 1-52.

3. K. R. Goodearl, von Neumann regular rings (Pitman, 1979).

Mathematical Institute

UNIVERSITY OF ST. ANDREWS

SCOTLAND
Department of Mathematical Science NORTHERN ILLINOIS UNIVERSITY De Kalb, IllinoIs 60115, U.S.A. 